\title{
Primary breast edema on contrast-enhanced digital mammography: a preliminary experience
}

\author{
Marwa A. Haggag ${ }^{1 *} \mathbb{D}$, Soha T. Hamed² and Aya Sedky Abd El Latif Mawas ${ }^{3}$
}

\begin{abstract}
Background: Primary breast edema can cause marked increase in skin thickness, breast density and echogenicity due to dense breast tissue filled with fluid and so causes subsequent significant attenuation of both the $x$-ray and ultrasound beams. The study aim is to assess the value of contrast-enhanced digital mammography (CEDM) in assessment and characterization of the obscured underlying breast lesions in cases of primary breast edema.

Results: Fifty five female participants were evaluated, of median age 51 years old and IQR 21. CEDM shows high sensitivity and specificity in the lesion detection as well as local extension delineation in cases associated with primary breast edema. It was accurate in detection of multifocal/multi-centric disease. CEDM is considered as a good negative test in cases of metastatic axillary lymph nodes to exclude and assess any associated obscured breast lesions, as it is good in delineating breast masses obscured by condensed parenchymal tissue. The calculated sensitivity of DM \& CEDM was 87.5\%, 95.8\%, specificity was 55.5\%, 72\%, the PPV and NPV were $91,93.6 \%$ and $45 \%, 77.8 \%$, respectively.
\end{abstract}

Conclusions: CEDM has an important additional diagnostic value in the assessment, characterization and better delineation of breast lesions in primary edematous breast cases.

Keywords: Contrast-enhanced digital mammography, Primary breast edema, Breast cancer, Dense breast

\section{Background}

Breast edema is common radiologic finding. It is associated with variety of mammary and extra mammary etiologies. Inflammatory breast carcinoma and infectious or non-infectious mastitis are two major reasons arising from breast itself [1]. The occurrence of edema significantly increased from benign over non-invasive to invasive breast cancer [2].

The mammographic findings of edematous breast changes are in form of increase skin thickness and increased parenchyma density as well as accentuated interstitial markings [3]. Breast edema in inflammatory breast cancer can cause tremendous skin thickening and breast enlargement that attenuates both the $x$-ray and

\footnotetext{
${ }^{*}$ Correspondence: marwa130140@yahoo.com

'Women's Imaging Unit, Department of Radiology, National Cancer

Institute, Cairo University, Giza, Egypt

Full list of author information is available at the end of the article
}

ultrasound beams because of the enlarged, dense breast tissue filled with fluid and tumor [4].

Contrast-enhanced digital mammography (CEDM) had a better diagnostic accuracy mainly due to improved specificity, and better positive and negative predictive values. CEDM could be useful in some cases demonstrating clinical or ultrasono-graphic abnormality with no obvious findings at mammography. It is particularly the case in dense breasts where normal breast tissue can hide a cancer [5].

CEDM adds functional information complementary to the morphologic findings of mammography (MX), aiding the lesions detection as well as characterization [6]. It is capable of detecting neo-angiogenesis and has the potential to play an important role in assessing multi-focality or multi-centricity in breast cancer [7].

Dual energy contrast-enhanced spectral mammography (DE- CESM) has the advantage of the assessment of functional information for both breasts after one contrast agent injection. It also provides immediate solution in 
the same mammography unit without delaying. In addition that no need for special technologist training as it is similar to standard mammography. DE- CESM has wide patient acceptance as it allows final decision at the end of the day. It is a fast technique that can be correlated easily with conventional mammograms. Also, radiologists can easily interpret the subtracted CEDM images and oncologist and the surgeons can easily understand them [8].

CEDM showed significant high lesion detection as well as accurate assessment of the local extent of the lesion in cases that is associated with breast edema. CEDM accurately assessed the multifocality/multi-centricity of the disease [9].

In this study, we aim to assess the contrast-enhanced digital mammography role in cases associated with primary breast edema and how it can help in detection and characterization of lesions. Its role as well and ability to assess the multifocality and multi-centricity of the disease.

\section{Methods}

\section{Patients}

This study is a prospective study. Fifty five patients presented with edematous breasts were included in the study. Standard digital mammography (DM) and CEDM were done for all patients. Cases were collected from December 2016 to December 2017 at the female imaging unit of the Radiology Department of the institute. All patients signed an informed consent that was approved by ethical committee (blinded for peer review).

The inclusion criteria included women presenting with breast edema on conventional imaging without any previous exposure to surgery or post chemotherapy or radiation to the same breast. The exclusion criteria included women that presented with breast edema after exposure to previous surgery, post radiotherapy, post chemotherapy, pregnant patients or patients with giving history of contraindication to IV contrasts material injection as allergy to contrast agents.

We correlated most breast lesions and pathological axillary lymph nodes with pathological results using surgery, excisional biopsy, skin punch biopsy, true cut biopsy, or fine needle aspiration cytology. Yet, follow-up were performed for some lesions classified as benign.

\section{Mammography system}

DE-CEDM was performed using GE Senographe 2000D full-field digital mammography system from GE Healthcare; Chalfont St-Giles, UK. (GE Healthcare) system with CEDM features.

Each CEDM view was done in form of pair of low and high energy $\mathrm{x}$-ray exposures in the same breast compression. Low energy image is similar to standard mammography and high energy image shows the contrast-enhanced areas that can help identify enhancing lesions.

\section{Technique of examination}

The examination starts with intravenous injection of an iodinated contrast agent (iohexol, $300 \mathrm{mg} \mathrm{I} / \mathrm{ml}$ ) at a dose of $1.5 \mathrm{ml} / \mathrm{kg}$ in a catheter introduced in the ante cubital vein (usually contra-lateral to the breast of concern). Then we apply compression, to avoid interference with the normal vascular dynamics of the breast. After $2 \mathrm{~min}$ wait, we started image acquisition. First: we performed $\mathrm{CC}$ (craniocaudal) view of the normal breast, then the two standard CC view and MLO (mediolateral oblique) view of the breast of concern. At the end we performed MLO view of the other breast. Degree of compression had to be at extent that can limit breast motion, but without affection and reduction blood flow. Low-energy images were acquired at 26 to 31 peak kilo voltage $(\mathrm{kVp})$ values which is below the k-edge of iodine $(33.2 \mathrm{keV})$. High-energy images were acquired at 45 to $49 \mathrm{kVp}$, which is above the k-edge of iodine. After image acquisition and through appropriate image processing, subtraction images become available in the four standard mammographic views. In this subtraction images, the visibility of normal parenchyma is reduced and we visualized almost only the abnormal enhancement.

\section{Image analysis}

In mammographic studies, lesions are assessed as usual (determining the size, site, shape, margin, definition, density and calcifications). In CEDM images, we can assess both low energy and subtracted images. We can identify non-enhancing suspicious clusters of micro calcifications and evaluate the morphologic features of non-enhancing mass lesions in low energy images. We can assess the presence or absence of contrast enhancement as well as pattern and degree of enhancement in the subtraction images. We depended in the interpretation of enhancing lesions on criteria of the dynamic MRI enhancement pattern interpretation according to kamal et al. [10].

\section{Statistical analysis}

We classified all inflammatory or negative cases of edematous breasts as benign for statistical analysis. In mammographic images, we assessed criteria as masses with suspicious characteristics, micro-calcific clusters or dense axillary lymph nodes as lesions with high probability of malignancy and classified as positive results. The sensitivity and specificity as well as PPV and NPV of mammography and CEDM were assessed. 


\section{Results}

\section{Classification of lesions}

The study included 57 edematous breast: 9/57 (15.7\%) were proven as benign and $48 / 57(84.3 \%)$ were proven as malignant (Fig. 1).

In our study, each breast together with its ipsilateral axillary lymph nodes managed as one unit (case) for statistical analysis to detect the causative lesion for edema. We correlated most breast lesions and pathological axillary lymph nodes with pathological results using surgery, excisional biopsy, skin punch biopsy, true cut biopsy, or fine needle aspiration cytology. Only three mastitis cases were followed up.

We classified all inflammatory edematous breasts as benign for statistical analysis. In mammographic images, we assessed criteria as masses with suspicious characteristics, micro-calcific clusters or dense axillary lymph nodes as lesions with high probability of malignancy and classified as positive results. Finally the sensitivity and specificity of MX and CEDM were compared.

Benign lesions included 9/57: 6/9 inflammatory (mastitis), one of them complicated by abscess, 3/9 inflammatory (granulomatous mastitis). While malignant breast lesions include 48/57cases as in (Table 1 ).

Multiple lesions were noted in 15/57 (26\%) edematous breasts. Twelve were pathologically proven as multicentric or multifocal invasive carcinoma. The other three cases were diagnosed pathologically as inflammatory (granulomatous mastitis).

Two cases were bilateral edematous cases that were proven as primary breast cancer in the contralateral one presented with marked edematous changes in mammography. The axilla was not exposed in both cases due

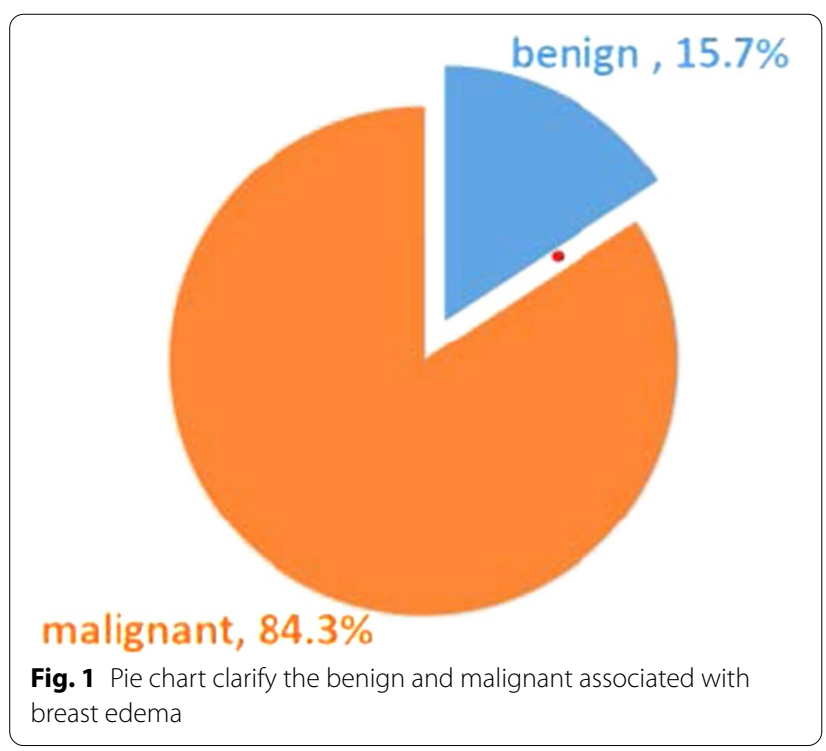

Table 1 Malignant breast lesions associated with edema and their final pathologic diagnosis

\begin{tabular}{lll}
\hline Pathologic results & No. of cases & Percentage \\
\hline Invasive duct carcinoma(IDC) & 31 & 64.6 \\
Invasive lobular carcinoma(ILC) & 4 & 8.3 \\
Invasive mammary carcinoma & 5 & 10.3 \\
$\quad$ (ductal, lobular) mixture & & \\
Ductal carcinoma insitu (DCIS) & 2 & 4.2 \\
Micro-papillary & 2 & 4.2 \\
Undifferentiated carcinoma & 2 & 4.2 \\
Mets adenocarcinoma & 2 & 4.2 \\
\hline
\end{tabular}

to defective mammographic technique, and so also contrast mammographic images but there was no enhancing breast lesions. Suspicious lymph nodes were seen by ultrasound and proven malignant by pathology.

\section{Standard mammography results}

Our cases have breast edema with variable degrees. Edema is evidenced by overall increased parenchymal density, accentuated trabeculation and increased skin thickness. Six cases presented with intense edematous changes obscured the margins of mammographically detected lesions and limit the ability of detection of multiplicity and satellites. Forty six cases showed positive mammographic findings in the form of asymmetry, micro-calcific clusters, and dense axillary lymph nodes as in (Table 2).

\section{CEDM results}

Enhancement was observed in 55/57 edematous breasts. No enhancement in two cases. 23/55 (41.8\%) cases showed non-mass enhancement (NME) and 27/55 (49.1\%) cases showed mass enhancement and 5/55 (9.1\%) cases included both enhancement types. The two nonenhancement cases show metastatic axillary lymph nodes as the cause of the edema which could not be detected by CEDM due to defective technique, but detected by ultrasound.

Table 2 shows initial mammographic findings

\begin{tabular}{ll}
\hline Initial mammographic findings & No. of cases \\
\hline Asymmetry & 40 \\
Micro-calcific clusters & 5 \\
Nipple retraction & 2 \\
Dense suspicious axillary lymph nodes & 15
\end{tabular}

N.B: there is more than one findings in some cases, e.g. asymmetry is associated with micro calcifications clusters in 3 cases 
Table 3 Correlation between distribution of non-mass enhancement (NME) by CEDM and final pathological findings

\begin{tabular}{|c|c|c|c|c|c|c|}
\hline Distribution & & Benign & & Malignant & & $P$ value \\
\hline Regional & 13 cases (46.4\%) & $8 / 9$ & $88.90 \%$ & $5 / 19$ & $26.30 \%$ & 0.011 \\
\hline \multirow[t]{2}{*}{ Segmental } & 2 cases (7.1\%) & & & & & \\
\hline & & 0 & 0 & $2 / 19$ & $10.50 \%$ & 1 \\
\hline Linear (ductal) & 8 cases $(28.6 \%)$ & 0 & 0 & $8 / 19$ & $42.10 \%$ & 0.05 \\
\hline Diffuse & 5 cases $(17.9 \%)$ & $1 / 9$ & $10.10 \%$ & $4 / 19$ & $21.10 \%$ & 1 \\
\hline \multirow[t]{2}{*}{ Total } & 28 cases & 9 & $100 \%$ & 19 & $100 \%$ & \\
\hline & $100 \%$ & & & & & \\
\hline
\end{tabular}

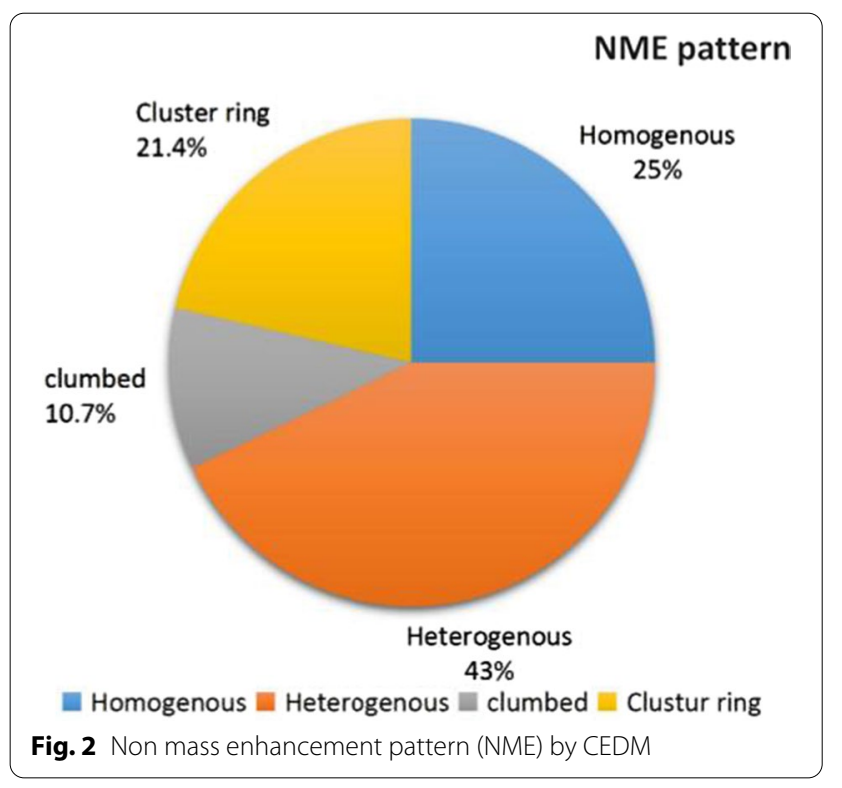

\section{Enhancement characteristics of the breast lesions}

Non-mass enhancement is further assessed according to the distribution pattern: regional, linear, segmental or diffuse was presented in (Table 3). CEDM images allows accurate and confident delineation of the ductal extension of mammographically detected lesion. NME is also classified according to internal enhancement characteristics as in (Figs. 2, 3). Non-mass enhancement is classified also according to degree of enhancement: 4 faint cases (14.2\%), 16 cases moderate cases (57.1\%) and 8 cases show intense enhancement (28.7\%). Nine cases of NME were benign and 19 cases were malignant, as proven pathologically.

Thirty two cases were enhancing masses. Mass enhancement was characterized according to: shape (regular, irregular), margin (circumscribed, ill defined, speculated), and internal mass enhancement characteristics as being homogeneous, heterogeneous, rim and septation as in (Table 4,5). Mass enhancement is

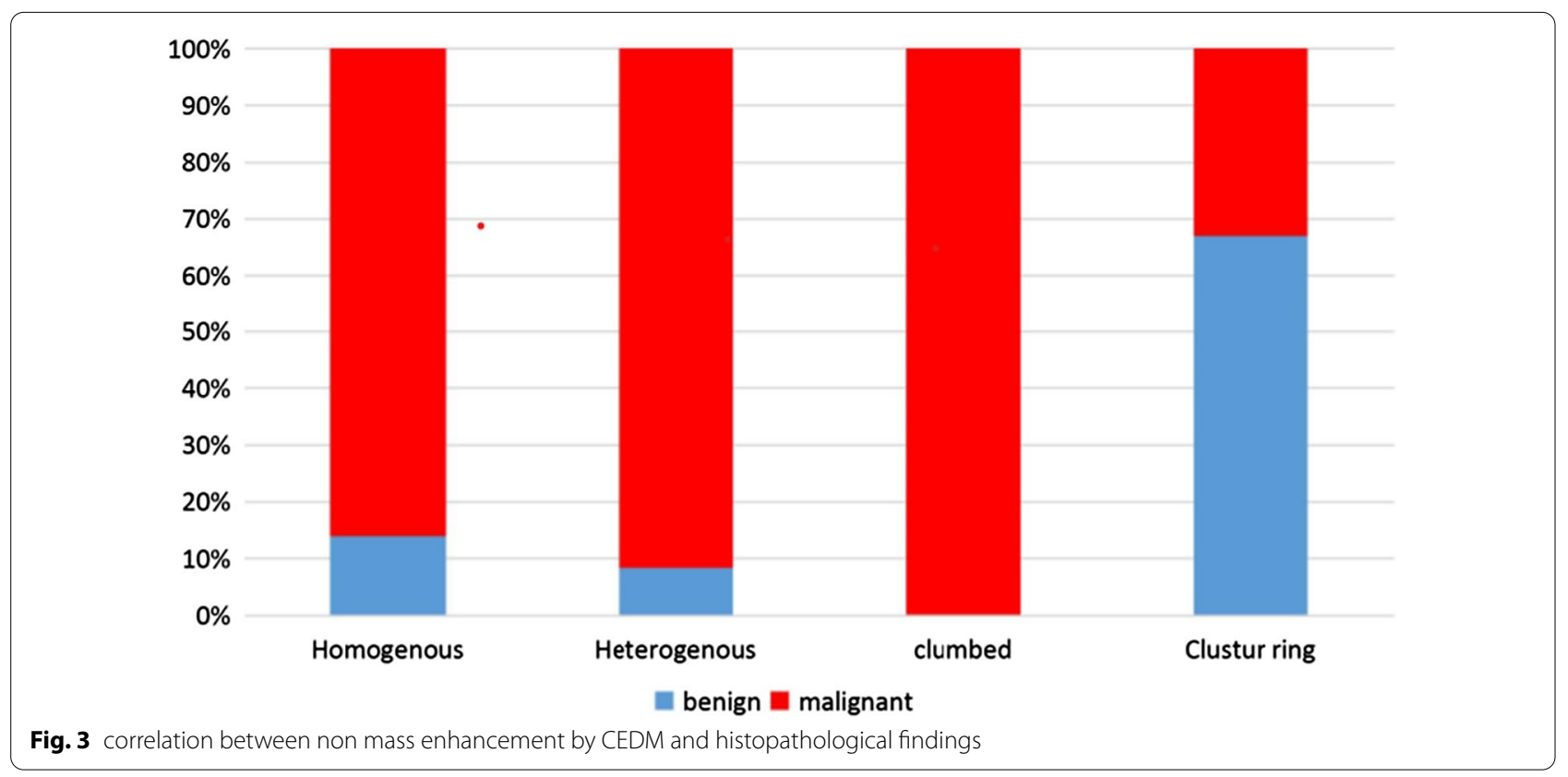


Table 4 Enhancing mass morphology by CEDM

\begin{tabular}{lll}
\hline Enhancing mass & Description & Count(32) \\
\hline Shape & Regular & 2 \\
& Obscured & 2 \\
Margin & Irregular & 28 \\
& Circumscribed & 1 \\
& Ill defined & 23 \\
& speculated & 8
\end{tabular}

Table 5 Mass enhancement pattern by CEDM

\begin{tabular}{lll}
\hline Mass internal enhancement & No of cases & Percentage \\
\hline Homogeneous & 2 & 6.25 \\
Heterogeneous & 28 & 87.5 \\
Rim & 2 & 6.25 \\
Total & 32 & 100 \\
\hline
\end{tabular}

classified also according to degree of enhancement: 4 faint cases (12.5\%), 16 cases moderate cases $(50 \%)$ and 12 cases show intense enhancement (37.5\%). All the 32 mass enhancing lesions, all proven pathologically as malignant lesions.

\section{Overall performance of CESM}

The calculated sensitivity, specificity, PPV and NPV of digital mammography were $87.5 \%, 55.5 \%, 91 \%$, and $45 \%$, respectively. The calculated sensitivity of DE-CESM was $95.8 \%$, specificity was $72 \%$, while the PPV and NPV were $93.62 \%$ and77.78\%, respectively as shown in (Fig. 4).

\section{Discussion}

Contrast-enhanced digital mammography (CEDM) is the only imaging modality that provides both high-resolution, low-energy images comparable to that of digital mammography and a contrast-enhanced image similar to that of magnetic resonance imaging. So CEDM has the potential to combine the relative ease, low cost, and the practicality of mammography with the high sensitivity of MRI [11].

Cheung et al. 2014 demonstrated that in women with dense breasts, CEDM has higher sensitivity and specificity compared to mammography, changed from $71.5 \%$ to $92.7 \%$ and from $51.8 \%$ to $67.9 \%$ respectively [12]

In our cases, mammographic low specificity was due to dense heterogeneous breast. Six cases presented with intense edematous changes obscured the margins of mammographic detected lesions and limit the ability of detection of multiplicity and satellites (Fig. 5). CEDM can delineate masses that are obscured by condensed parenchyma and provide higher sensitivity and specificity.

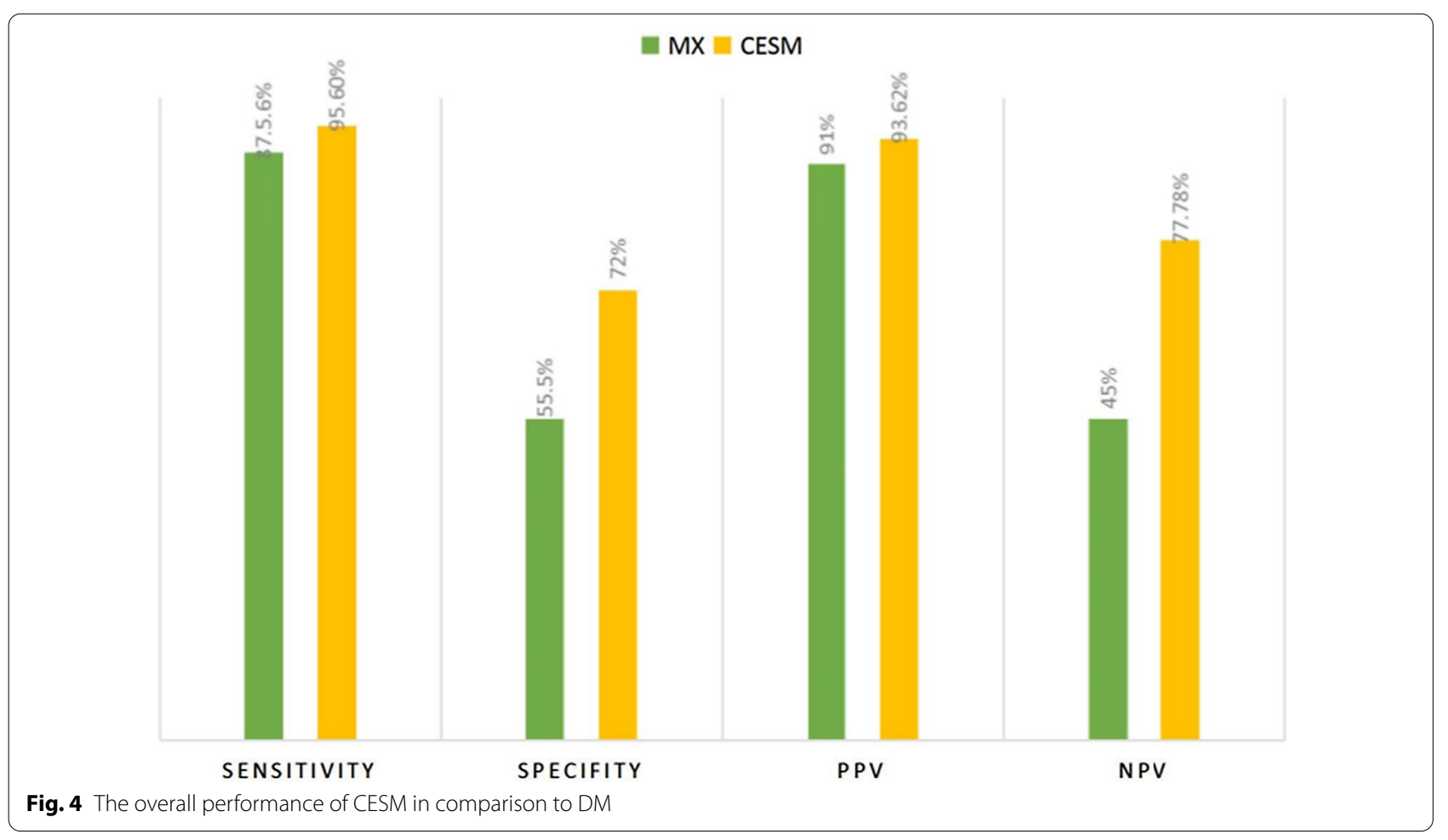




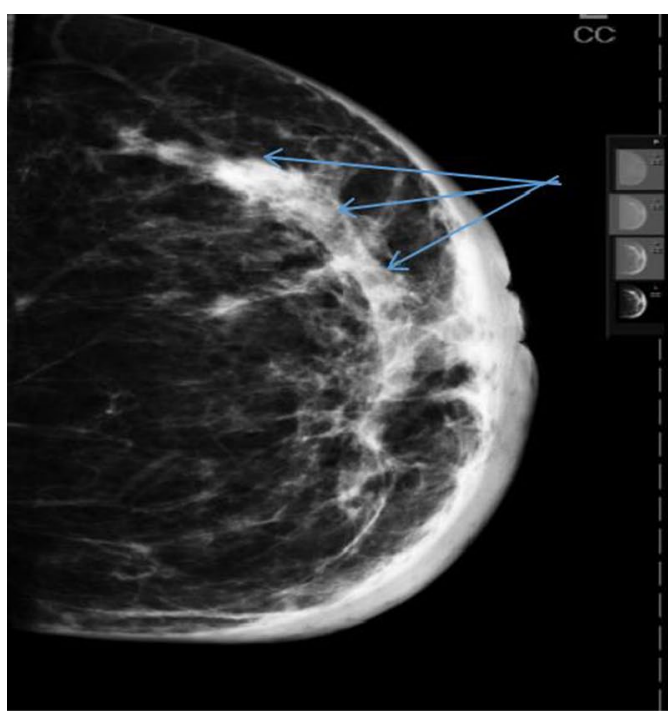

A

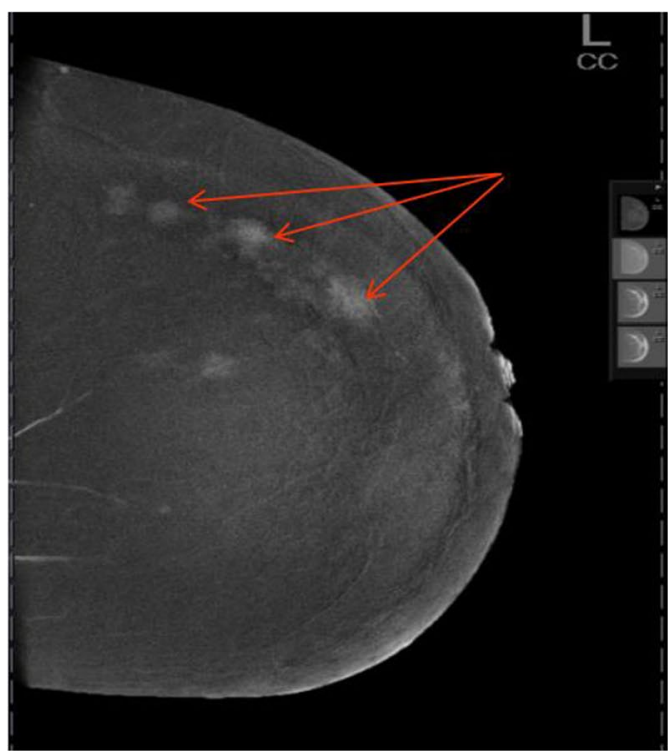

C

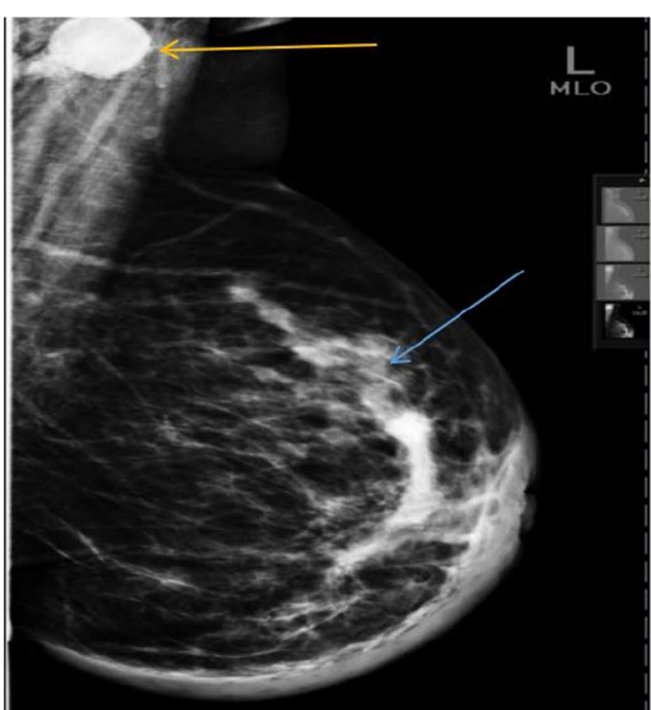

B

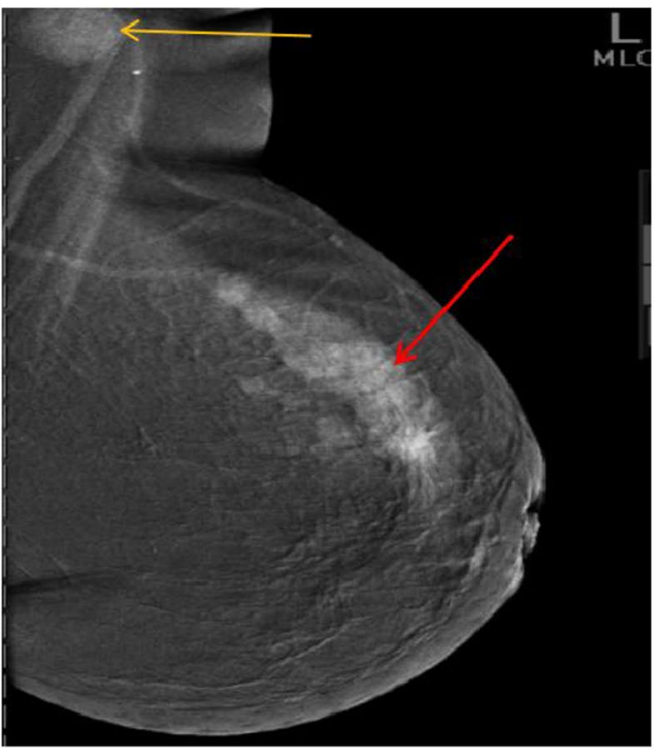

D

Fig. 5 A 47-year-old female patient complains of left primary breast edema. $\mathbf{a}, \mathbf{b} \mathrm{DM}, \mathbf{a} C \mathrm{C}$ and $\mathbf{b}$ MLO views of the left breast showed moderate edema pattern with multiple upper outer quadrant (UOQ) ill-defined focal asymmetrical lesions (blue arrows) and left suspicious ipsilateral axillary lymph node LN (yellow arrow). c, d CEDM c CC and $\mathbf{d}$ MLO views of the left breast showed UOQ multifocal enhancing speculated masses (red arrows). Pathological diagnosis: Left breast IDC, Grade 2

Elsaid et al. 2015 performed a prospective study on 34 female patients with breast edema and concluded that DE-CEDM is able to demonstrate lesions that cannot be detected by mammography and also is a promising modality in follow-up of cases. The calculated sensitivity of DE-CEDM compared to the digital mammography compared was $92 \%$, versus $74 \%$, specificity of $100 \%$, compared to $77 \%$. [13].
While our inclusion criteria of the study were limited to primary breast edema cases. Our results showed significant increase in sensitivity of CEDM compared to MX alone. The calculated sensitivity of CEDM vs MX was $95.8 \%$ vs $91.49 \%$, calculated specificity was $70 \%$ vs $50 \%$.

In our study, we concluded that CEDM can confidently exclude breast lesions that could be obscured in edematous parenchyma in pathological proven 
metastatic axillary lymph nodes (2 cases), so considered as good negative test. CEDM can delineate masses that are obscured by condensed parenchyma, so considered as a good positive test. MX alone can detect abnormality in 42/57 malignant breast edema, compared to $46 / 57$ detected by CEDM. In CEDM only two false negative cases, edema was caused by lymphatic obstruction secondary to nodal metastasis (that was missed due to defective technique).

In our study, retro areolar lesions could be easily masked as breast tissue is denser in this region especially due to edema and CEDM could demonstrate any obscured underlying lesion. Ten cases were detected as retro-areolar focal asymmetrical density. Four were proven as IDC, 2 cases were invasive mammary, and 4 cases were periductal mastitis / granulmatous mastitis as proven pathologically.

CEDM could clearly delineate the ductal extension for mammographic detected lesions. In our study, ductal extension were seen in 8 cases all detected by CESM (100\%).

Also Elsaid et al. 2015 concluded that CEDM can accurately delineate and assess multi-focality/multi-centricity disease in 5/14 cases with edematous breasts. While only 1 and 3 cases detected by MX alone and US respectively [13]. In our study, a speculated lesion is visible on mammography, multifocality was established on CEDM. Among the 15 patients with multifocal /multi-centric 12 histologically proven IDC lesions and 3 proven granulmatous mastitis, all were detected by CEDM versus ( 5 cases only) detected as multifocal/multicenteric by MX alone.

For cases with bilateral multiple lesions, it is not practical to underwent biopsy from all the lesions. So, lesions that have the most abnormal and suspicious contrast uptake on CEDM are the best to be targeted for histopathological evaluation and thus can help in triaging lesions.

We consider non-contrast uptake, diffuse parenchymal uptake as probably benign, while ring enhancement was observed in both benign and malignant lesions. In our study, no enhancement was seen in two cases. Edema was caused by lymphatic obstruction secondary to nodal metastasis (that were missed due to defective technique), considered as two false negative cases (Table 6).

In our study, rim enhancement was detected in 2 cases. Both cases proven histo-pathologically being malignant, so should not conclusively categorized as benign lesion. On the other hand, clustered ring NME should not be conclusively categorized as malignant lesions. In our study, 6 cases were detected by CEDM 5 proven to be benign as inflammatory granulomatous mastitis or mastitis (Fig. 6), and 1 was proven to be malignant as invasive
Table 6 False positive and false negative cases on CEDM

\begin{tabular}{lll}
\hline False diagnosis & Pathologic diagnosis & \\
\hline False negative & & 2 \\
False positive & Mets undifferentiated carcinoma & 2 \\
& & 3 \\
& Granulmatous mastitis & 2 \\
& Mastitis & 1 \\
\hline
\end{tabular}

lobular carcinoma (Fig. 7). So in these two patterns of enhancement, correlation with US is important. This coincides with kamal et al. 2016, that concluded that rim enhancement and clustered ring NME cases need additional diagnostic modality such as ultrasound to be coupled with CEDM results and should not be categorized alone as malignant lesions.

In our study, non-mass distribution as segmental and linear showed a high prediction probability of malignancy as 2 cases showed as segmental, 8 cases showed as linear all are proven as malignant. Also in our study, non-mass internal enhancement pattern as clumped pattern ( 3 cases were detected all proven to be malignant) and heterogeneous pattern as ( 9 of 12 cases proven to be malignant) and so showed high prediction probability of malignancy. In accordance with kamal et al. 2016 who stated that enhancement with high malignant probability included segmental, ductal and regional clumped or heterogeneous enhancement.

In our study, we concluded also that mass enhancement of high malignant probability included irregular-shaped enhancing mass lesions with speculated and irregular margins and heterogeneous enhancement.

Luczyńska et al. 2016 study revealed that all invasive and intraductal carcinomas showed enhancement on CEDM. They states that $83 \%$ of the invasive carcinomas mostly showed intense and moderate enhancement on CEDM, while $87.5 \%$ of intraductal carcinoma cases show weak enhancement [14]. In our study, intense contrast enhancement on CEDM shows high malignant probability as (17/20 intense enhancing cases) has proven pathologically to be malignancy, yet the rest (3 cases) are inflammatory process showing micro abscess with granulomatous formation.

Also in our study, (5 cases) with subtle suspicious clustered micro-calcifications without mammographically visible underlying lesion, $4 / 5$ of those cases were pathologically intraductal carcinoma while the fifth was invasive lobular cancer. Three cases showed mammographically masses with clustered suspicious micro-calcification all of them pathologically. All the 8 cases showed faint to moderate enhancement on CEDM (only one of them was faint). 


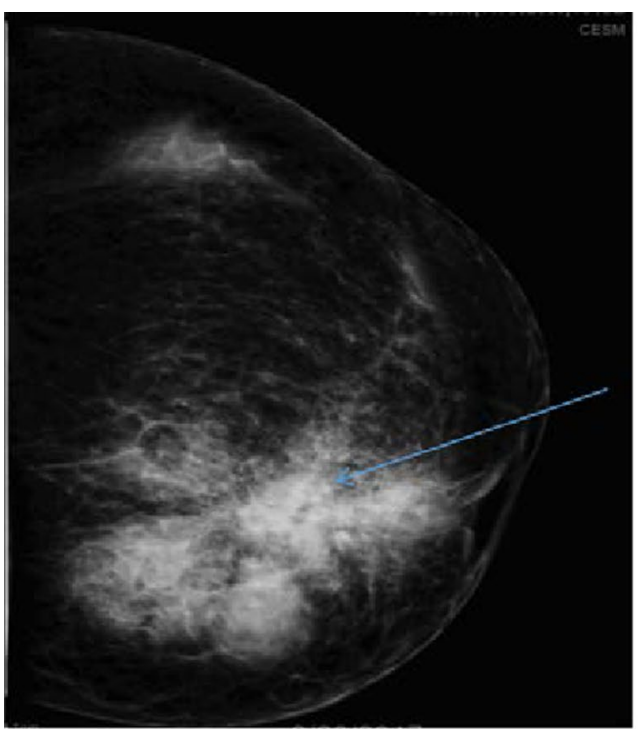

A

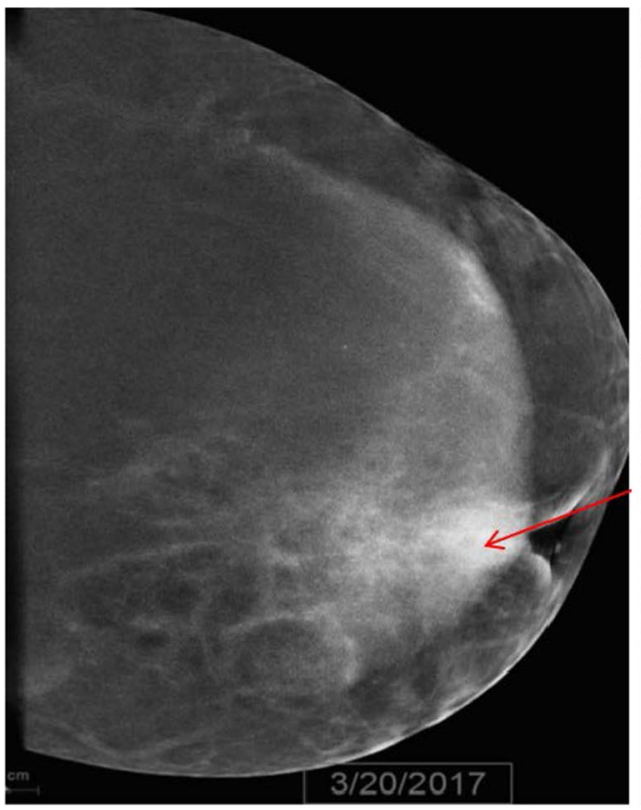

C

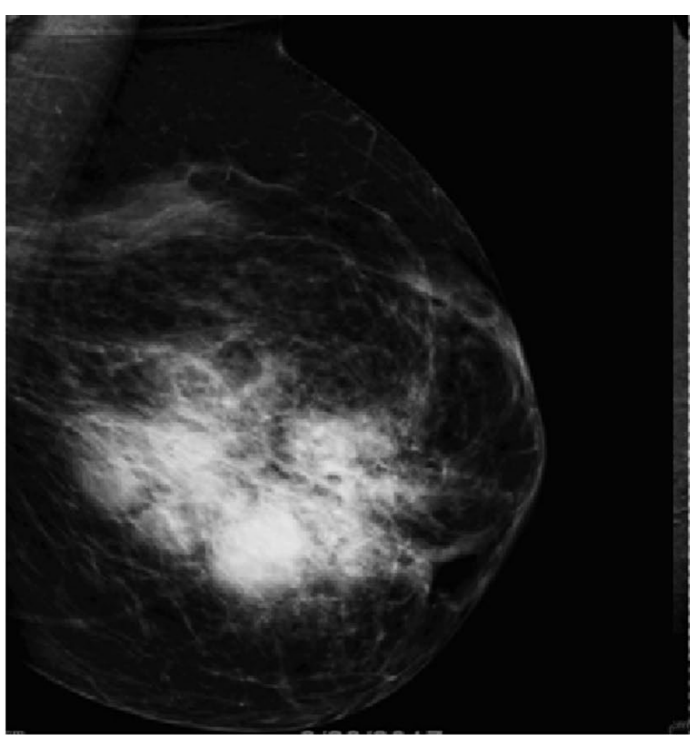

B

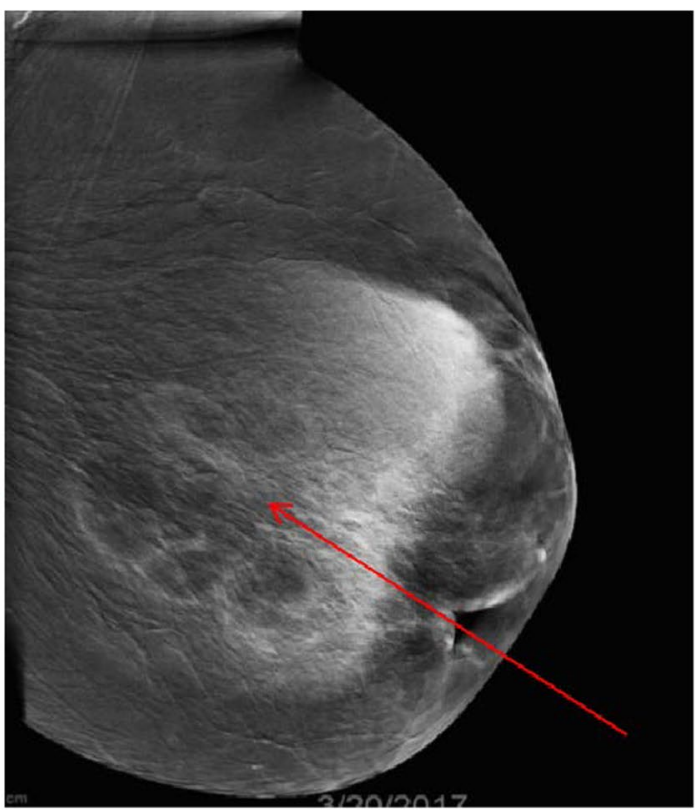

D

Fig. 6 A 40-year-old female patient complains of left primary breast edema. a, b DM, a CC and b MLO views of the left breast showed minimal focal edema pattern in the form of skin thickening, accentuated trabeculation, increased over all density. Retro-areolar dense ill-defined mass with architectural distortion encroaching LIQ (blue arrow). c, d CEDM: c CC and $\mathbf{d}$ MLO views of the left breast showed moderate multiple clustered ring enhancing lesions at the retro-areolar region with parenchymal enhancement (red arrows). Pathological diagnosis: Periductal granulomatous mastitis

Knogler et al. 2017 revealed that the enhancement characteristics of the malignant lesions are similar in both CEDM and MRI. Their study has included mass enhancement only, yet believed that non-mass enhancement in CEDM could be based also on the MRI BI-RADS descriptors for non-mass enhancement [15].
We have already depend on MRI BI-RADS descriptors in assessment of both mass and non-mass enhancing lesions according to kamal et al. 2016 and we believed that we can differentiate between benign and malignant lesions depending on the application of the MRI BIRADS descriptors in CEDM. Yet, further large group 


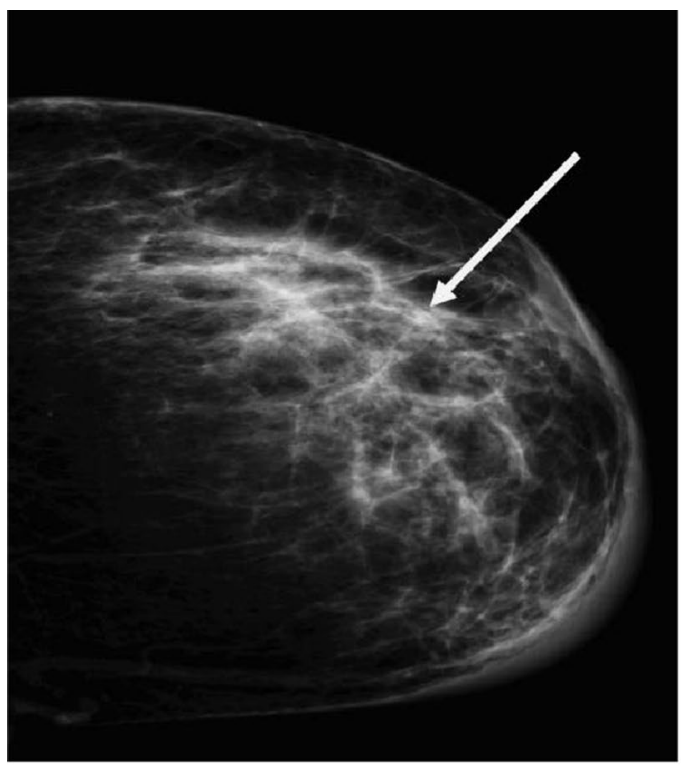

A

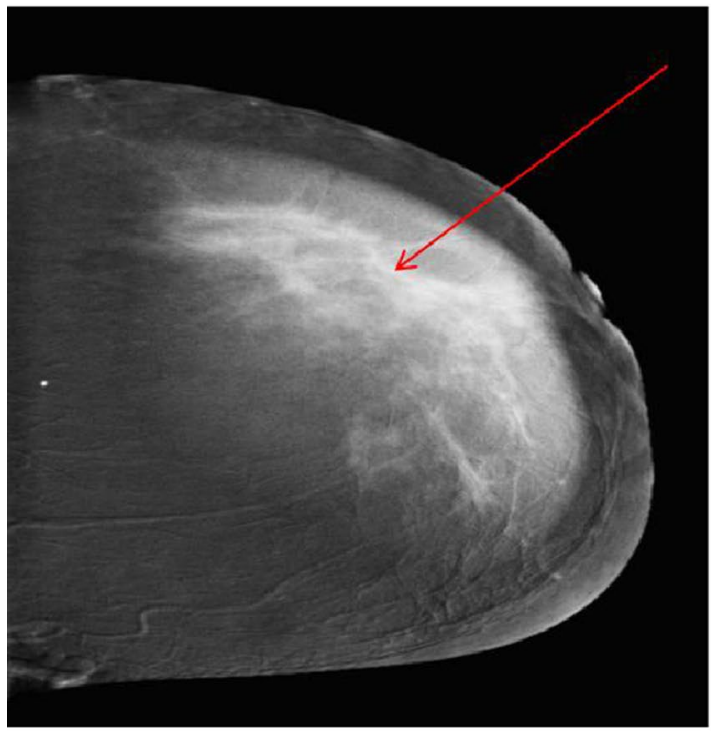

C

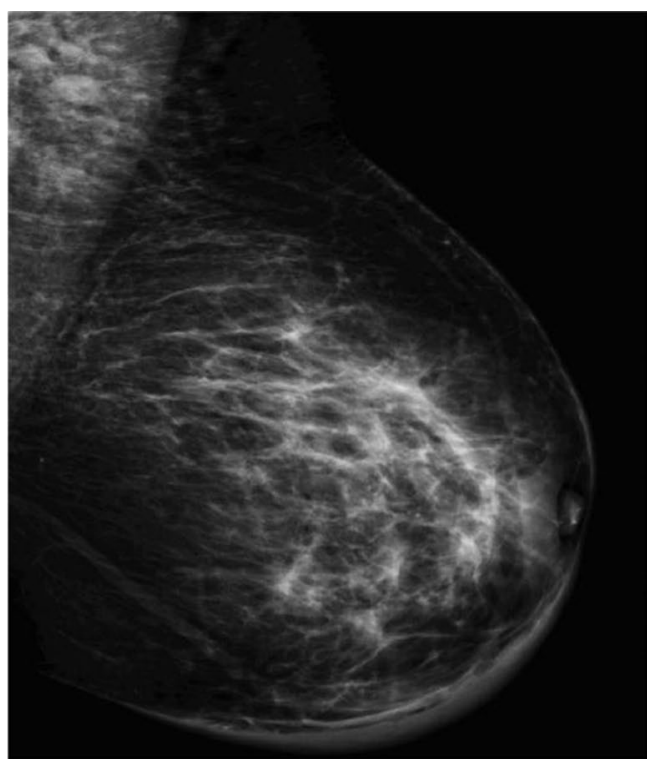

B

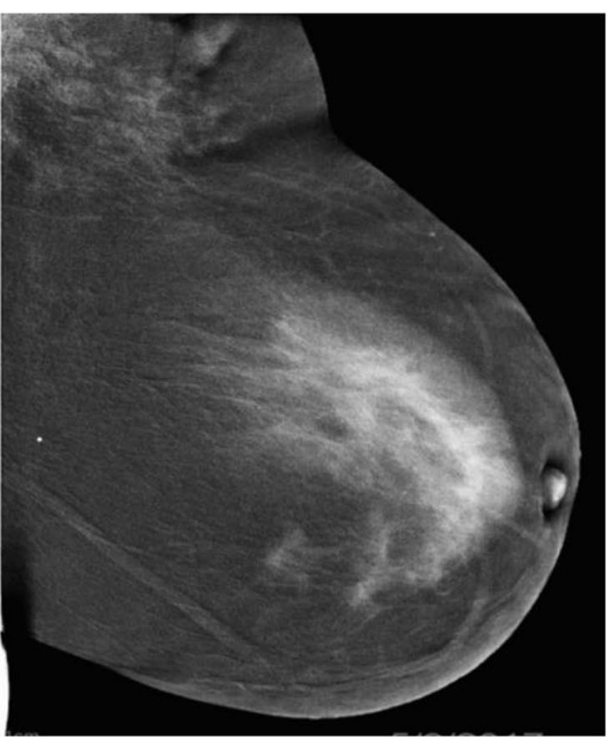

D

Fig. 7 A 63-year-old female patient, complains of left primary breast edema. $\mathbf{a}$, $\mathbf{b}$ : DM $\mathbf{a}$ CC and $\mathbf{b}$ MLO views of the left breast showed moderate edema pattern in the form of skin thickening, accentuated trabeculation. UOQ architectural distortion (white arrow). c, $\mathbf{d}$ CEDM: c CC and $\mathbf{d}$ MLO views of the left breast showed moderate heterogeneous non-mass cluster ring enhancement UOQ encroaching on LQ.(red arrows). Pathological diagnosis: Invasive lobular Carcinoma

studies involving non-mass enhancement as well are essential.

\section{Limitation of our study}

First: we had higher malignancy proportion in our patient sample because we had conducted the study in oncology institute. Second: the number of patients included in the study is still small. Third: no standardized lexicon for morphological enhancement characterization in CEDM, like that of the 5th edition of the ACR BI-RADS Atlas on DCE-MRI. Still it is essential to perform a standardized lexicon of morphology descriptors and analyzing enhancing lesions identified on CEDM. The real specificity of CEDM is still unclear. 


\section{Recommendation}

Further larger studies that is including larger numbers and including mass and non-mass lesions are essential.

\section{Conclusions}

The present study confirms that is CEDM is a good problem solving technique in identification of lesions especially in dense as edematous breasts and it shows good assessment of multi-centricity/multifocality and ductal extension. Also CEDM allows accurate delineation of masses that could be obscured by dense parenchyma. It shows significant increase of the sensitivity without loss in specificity compared to mammography alone. It shows increase in the negative predictive value and subsequent significant decrease in the false negatives.

\section{Abbreviations}

CEDM: Contrast-enhanced digital mammography.; DE-CEDM: Dual energy contrast-enhanced digital mammography.; NME: Non mass enhancement.; MX: Mammography; CC: Craniocaudal; MLO: Mediolateral oblique; LN: Lymph node.; UOQ: Upper outer quadrant.

\section{Acknowledgements}

Not applicable.

\section{Authors' contributions}

MAS collected, analyzed and interpreted the patient images and patients information as well as pathological results. HMA suggest the idea, shares in collection, analysis and interpretation of the patient images, and manuscript preparation. STH supervises and do final interpretation of the patient images. All authors read and approved the final manuscript."

\section{Funding}

Not applicable.

\section{Availability of data and materials}

The datasets used and/or analysed during the current study are available from the corresponding author on reasonable request.

\section{Declarations}

\section{Ethics approval and consent to participate}

Approval for this study was obtained from the Research Ethics Committee of Kasr El Ainy Hospital, Cairo university. All study procedures were carried out in accordance with the Declaration of Helsinki regarding research involving human subjects. The committee's reference number is not available at this time. All patients included in this research gave written consent to participate in this research.

\section{Consent for publication}

Not applicable.

\section{Competing interests}

The authors declare that they have no competing interests.

\section{Author details}

${ }^{1}$ Women's Imaging Unit, Department of Radiology, National Cancer Institute, Cairo University, Giza, Egypt. ${ }^{2}$ Women's Imaging Unit, Department of Radiology, Kasr El Ainy Hospital, Cairo University, Giza, Egypt. ${ }^{3}$ M.B.B.CH Faculty of Medicine, Cairo University, Giza, Egypt.

Received: 13 February 2021 Accepted: 11 August 2021

Published online: 24 August 2021

\section{References}

1. Okcu, I. BILGEN, A. Oktay (2016). Breast edema related to extramammary disease: etiology and radiologic findings. ECR

2. Baltzer PA, Yang F, Dietzel M, Herzog A, Simon A, Vag T, Kaiser WA (2010) Sensitivity and specificity of unilateral edema on T2w-TSE sequences in MR-mammography considering 974 histologically verified lesions. Breast J 16(3):233-239

3. An YY, Kim SH, Cha ES, Kim HS, Kang BJ, Park CS, Yoon SK (2011) Diffuse infiltrative lesion of the breast: clinical and radiologic features. Korean J Radiol 12(1):113-121

4. Ikeda DM (2011) Breast imaging: the requisites. Mosby, Elsevier, Amsterdam

5. Dromain C, Thibault F, Muller S, Rimareix F, Delaloge S, Tardivon A, Balleyguier C (2011) Dual-energy contrast-enhanced digital mammography: initial clinical results. Eur Radiol 21(3):565-574

6. Dromain C, Thibault F, Diekmann F, Fallenberg EM, Jong RA, Koomen M, Toledano A (2012) Dual-energy contrast-enhanced digital mammography: initial clinical results of a multireader, multicase study. Breast Cancer Research 14(3):565-574. https://doi.org/10.1186/bcr3210

7. Daniaux M, De Zordo T, Santner W, Amort B, Koppelstätter F, Jaschke W, Marth C (2015) Dual-energy contrast-enhanced spectral mammography (CESM). Arch Gynecol Obstet 292(4):739-747

8. Muller S, Dromain C, Balleyguier C, Patoureaux F, Puong S, Bouchevreau X (2010) Contrast enhanced digital mammography (CEDM): from morphological to functional mammography. Poster No. C-0300, ECR

9. Mokhtar O, Mahmoud S (2014) Can contrast enhanced mammography solve the problem of dense breast lesions? Egpt J Radiol Nucl Med 45(3):1043-1052. https://doi.org/10.1016/j.ejrnm.2014.04.007

10. Kamal RM, Helal MH, Mansour SM, Haggag MA, Nada OM, Farahat IG, Alieldin NH (2016) Can we apply the MRIBI-RADS lexicon morphology descriptors on contrast-enhanced spectral mammography? Br J Radiol 89(1064):20160157

11. Lewis TC, Pizzitola VJ, Giurescu ME, Eversman WG, Lorans R, Robinson KA, Patel BK (2017) Contrast-enhanced digital mammography: a singleinstitution experience of the first 208 cases. Breast J 23(1):67-76

12. Cheung YC, Lin YC, Wan YL, Yeow K, M\& Chang, C. J. (2014) Diagnostic performance of dual-energy contrast-enhanced subtracted mammography in dense breasts compared to mammography alone: interobserverblind- readinganalysis. Europeanradiology 24(10):2394

13. EISaid NAE, Farouk S, Shetat OMM, Khalifa NM, Nada OM (2015) Contrast enhanced digital mammography: Is it useful in detecting lesions in edematous breast? Egypt J Radiol NuclearMed 46(3):811-819

14. Łuczyńska E, Niemiec J, Hendrick E, Heinze S, Jaszczyński J, Jakubowicz $J$ (2016) Degree of enhancement on Contrast Enhanced Spectral Mammography (CESM) and lesion type on Mammography (MG): comparison based on histological results. Med Sci Monit 22:3886

15. Knogler T, Homolka P, Hoernig M, Leithner R, Langs G, Waitzbauer M, Helbich TH (2017) Application of BI-RADS descriptors in contrastenhanced dual-energy mammography: comparison with MRI. Breast Care 12:212-216. https://doi.org/10.1159/000478899

\section{Publisher's Note}

Springer Nature remains neutral with regard to jurisdictional claims in published maps and institutional affiliations. 